\title{
Resultados del tratamiento hiposensibilizante en niños con asma bronquial. Comunicación preliminar
}

Dres. Gonzalo Gómez *, Sergio lbáñez* y Víctor Lama

Está demostrado dentro de la etiopatogenia del asma bronquial como factor importante la existencia de un terreno atópico, ligado, por una parte, a un estado de hiperreactividad bronquial y por otra, al desarrollo de una hipersensibilidad de tipo I, mediado por anticuerpos reagíni$\cos$, a diversas sustancias especialmente inhalantes, que corrientemente son inocuas para el común de la especie humana. Las bases actuales de tratamiento de hiposensibilización hacen que esté indicado para tratar los estados de hipersensibilidad frente a alergenos imposibles de evitar (polvo de habitación, hongos, pólenes) ${ }^{1-}$ 2. 3-4-5-6-7-b-0-10-11-12..13-14-16-16-17-19-14-20-21-22-25-2627

Hacemos notar el escaso número de niños

*Departamento de Pediatria, Area Oriente, Hospital Luis Calvo Mackenna. asmáticos que habitualmente reciben tratamiento hiposensibilizante, en parte debido a la creencia de que una gran proporción de estos nin̄os curan espontáneamente durante la adolescencia $^{24}$; por otra parte, el escepticismo que muchos padres y médicos tienen de la efectividad del tratamiento.

La presente comunicación informa de los resultados obtenidos en nuestro servicio desde el 15 de junio de 1969, en niños portadores de asma perenne, al cabo de 3 años de inyectoterapia y que fueron evaluados durante un año de observación posterior.

\section{MATERIAL Y METODO}

Nuestro material está constituido por 38 niños con asma bronquial perenne, test de alengia positivo y con una edad tope de 12 años. Ingresados 
desde el 15 de junio de 1969 y que al 15 de junio de 1973 habian cumplido 3 años de inyectoterapia a lo menos, realizándose la evaluación de los casos durante el tercer año.

Ya, en una comunicación previa recientemente publicada ${ }^{2 \theta}$, se hizo una relación del criterio de admisión y de estudio de nuestros pacientes asmáticos.

En cuanto a la evaluación de la severidad del cuadro asmático se usó el criterio de Johnstone, basado en el número de días de jadeo espiratorio por año (asma leve: hasta 10 días, moderado 11 a 70 días y severo más de 70 días) ${ }^{23 \cdot 24}$

En todos estos casos se indicó tratamiento hiposensibilizante con alergenos del Laboratorio Center, de acuerdo a los resultados de reactividad cutánea del test de alergia ${ }^{28-2 *}$, que se efectuó intradérmico con polvo habitación, mezcla de hongos (Alternoria, Hormodendrum, Aspergillus y Penicillium) y mezcla de pólenes (fresno, abedul, nogal, olmo, arce mojo, roble, álamo, plátano oriental, sauce, alfalfa, pasto azu, maiz, trébol, pasto forrajero, trigo Diente de León, Siete venas, artemisa y acederilla) a concentración de 1.000 P.N.U. por cc, de acuerdo a las especificaciones de dicho laboratorio, y usando como eslabón un control intradérmico de solución salina fisiológica fenolada al $0,5 \%$. Se consideró positivo toda reacción una cruz o más (cada cruz equivale a $5 \mathrm{~mm}$ de edema y eritema).

Para tratamiento se empleó mezcla de alergenos a los cuales el paciente manifestó positividad, se inició con una dosis de 0,05 ce de solución de 100 P.N.U. de cada alergeno, con incremento semanal durante 6 semanas, posteriormente con frecuencia bisemanal durante 2 meses para continuar con inyectoterapia mensual hasta el momento del control. Las dosis en cuanto a volumen y concentración se incrementaban hasta obtener la dosis máxima tolerada (aparición de molestias asmáticas después de la inyectoterapia y/o aparición de molestias locales intensas). Se mantuvo una dosis inmediatamente inferior a la que provocó dichas reacciones.

\section{RESULTADOS}

1. Relación de resultados de la inyectoterapia y sexo de los pacientes:
Tabla 1

\begin{tabular}{lcc} 
& Fementno & Mascuino \\
Pierde asma & 15 & 12 \\
Persiste asma & 4 & 7 \\
Total & 19 & 19 \\
\hline
\end{tabular}

En esta tabla se observa que no existe una diferencia significativa entre ambos sexos.

2. Resultados de la inyectoterapia relacionados con la edad de comienzo de las materias:

Tabla 2

\begin{tabular}{lccc}
\hline & $\begin{array}{c}\text { Menos de } \\
12 \text { añof }\end{array}$ & $\begin{array}{c}\text { De } 2 \text { a } 5 \\
\text { años }\end{array}$ & bo más años \\
\cline { 2 - 4 } & $\mathrm{B}$ & 13 & 6 \\
Pierde asma & 3 & 6 & 2 \\
\hline & 11 & 19 & 8 \\
\hline
\end{tabular}

En los diversos grupos no se aprecia una diferencia significativa.

3. Resultados de la inyectoterapia en relación al tiempo de evolución de la enfermedad:

Tabla 3

\begin{tabular}{lcc}
\hline & $\begin{array}{c}\text { Menos de jaños } \\
\text { enfenedad }\end{array}$ & $\begin{array}{c}30 \text { mas años } \\
\text { exfemedad }\end{array}$ \\
\cline { 2 - 3 } & 12 & 15 \\
Pierde asma & 5 & 6 \\
\hline
\end{tabular}

En los dos grupos se observa un resultado similar.

4. Resultados de inyectoterapia en rełación a antecedentes familiares de asma bronquial:

Tabla 4

\begin{tabular}{lcc}
\hline & \multicolumn{2}{c}{$\begin{array}{c}\text { Con antecedente } \\
\text { de asma familear de asma fomiliar }\end{array}$} \\
\cline { 2 - 3 } & 16 & 11 \\
Pierde asma & 7 & 4 \\
Persiste asma & 23 & 15 \\
\hline
\end{tabular}

Notamos resultados semejantes en ambos grupos. 
5. Relación resultado de inyectoterapia con grado de positividad del test de alergia:

Tabla 5

\begin{tabular}{lcc}
\hline & $+t++$ & $+++1++++$ \\
\cline { 2 - 3 } Pierde asma & 18 & 9 \\
Persiste asma & 5 & 6 \\
\hline rotal & 23 & 15 \\
\hline
\end{tabular}

De la presente tabla podría concluirse que aquellos pacientes con reacciones intensas al test de alergia intradérmico presentan una menor respuesta al iratamiente desensibilizante, pero dado lo reducido de nuestra casuística no nos atrevemos a formular una conclusión al respecto.

6. Resultados de inyectoterapia. Evaluación durante tercer año. 38 casos:

Tabla 6

\begin{tabular}{lcc}
\hline & Nimero & Porcentoje \\
\cline { 2 - 3 } & 27 & 71 \\
$\begin{array}{l}\text { Pierde asma } \\
\text { Pierde asma menor in- } \\
\text { tensidad } \\
\begin{array}{c}\text { Persiste asma igual in- } \\
\text { tensidad }\end{array}\end{array}$ & 10 & 26,3 \\
Empeoraroiento & 1 & 2,6 \\
Total & 0 & 0 \\
\hline
\end{tabular}

7. Relación de resultados de inyectoterapia con la severidad de las manifestaciones asmáticas:

Tabla 7

\begin{tabular}{|c|c|c|c|}
\hline & Asma leve & $\begin{array}{c}\text { Asma } \\
\text { moderado }\end{array}$ & Asma severo \\
\hline Pierde asma & 8 & 14 & 5 \\
\hline Persiste asma & 0 & 8 & 3 \\
\hline Total & 8 & 22 & 8 \\
\hline
\end{tabular}

En las formas leves la mejoria con el tratamiento desensibilizante es mayor.

\section{COMENTARIO Y CONCLUSIONES}

De la presente comunicación se desprende que con tratamiento desensibilizante, usando una dosis máxima tolerada, se consigue la desapari- ción de las molestias asmáticas durante el $3^{\text {er }}$ año de tratamiento en el $71 \%$ de los pacientes. Porcentaje que consideramos satisfactorio. Johnstone ${ }^{23}$, en su experiencia de 4 años de observación encuentra $70 \%$ de éxito en 2 grupos tratados: pero en el grupo en que se usó la dosis máxima el éxito fue de un $81 \%$. El mismo autor $^{24}$, en un estudio comparativo en $210 \mathrm{ni}$ ños seguidos hasta los 14 años de edad, encuentra un exito de $76 \%$ en el grupo tratadio con dosis máximas toleradas.

No encontramos una relación entre el resultado de la inyectoterapia con: sexo, edad de comienzo de las molestias asmáticas, tiempo de evolución de la enfermedad al momento de iniciar el tratamiento y antecedentes de asma familiar. Esto está de acuerdo con lo publicado con otros autores ${ }^{24}$.

Existiría un mejor resultado en el grupo de paciente portador de formas leves. Debido a lo reducido de nuestra casuistica creemos que este dato había que investigarlo más a fondo, tanto más, cuando en otras publicaciones se ha encontrado algo diferente ${ }^{24}$.

De un punto de vista operacional, considerando que nuestros pacientes son a veces niños de corta edad, y que por lo tanto hay limitaciones anamnésticas y de técnica, creemos que es de utilidad el empleo de mezcla de alergenos en el test.

Nos parece que nuestro esquema de tratamiento semanal durante 6 semanas, bisemanal durante 2 meses, seguido por una dosis mensual, hasta completar el tratamiento, es fácil de ser cumplido.

\section{RESUMEN}

Se comunican los resultados obtenidos con inyectoterapia desensibilizante enıre 38 nin̄os asmáticos perenne, tratados durante 3 años y evaluados en el $5^{\prime}$ año.

Se obtiene la desaparición de las molestias asmáticas durante codo el $3^{\prime \prime}$ año en el $71 \%$ de los casos. Se usó una dosis máxima tolerada.

No se encontró relación evidente, entre el resuliado del ratamiento con: sexo, edad de comienzo de las molestias asmáticas, tiempo de evolución de la enfermedad al momento de iniciar el tratamiento y antecedentes de asma en la familia.

Se destaca la utilidad operacional del uso de mezcla de ajergenos en el test.

Queda planteado un esquema de tratamiento. 


\section{SUMMARY}

38 perennial asthmatic children were treated for 3 years with hiposensitization therapy. Results after treatment are presented, $71 \%$ of children had complete remission of asthmatic simptoms during the whole third year of ireatment.

The highest tolerated dose was used.

No relationship was found between the result of treatment and sex, age at onset of simptoms, duration of jllness. or asthma in relatives.

The use of allergens mixtures for the allergic test was mostly useful.

A therapeutic scheme is presented.

\section{REFERENCIAS}

1 Margoret Tumer. WasWick. Medicine 14:889, 1974 .

2 Frandsen, S. Acta allengol. 12:341, 1958.

s Kendig, E.L., jr. Disorders of the Respiratory Tract in Children, Philadelphia W.B. Saunders, 1967.

- Gómez, Gonzalo; loáriez, Sergio. Rev. Chilena Pediatría, Vol. 45:88, enero-lebrero 1974.

s H. Mansmann, jr. The Ped. Clinics of N.A. 15:357, 1967

- Milman, B.C. Ann of Allerg. 25:620, 1967.

7 Kantor and Speer. "Characteristics of the allergic child", The Allergic child. Ed. por Speer, Hoebel, New York, 1963.

- Hamen, $K$. AAS at ad. Arch. of Discase in Child. $48: 850,1973$.

- L. Kumar; R.W. Neucomb; K. Ishezaka; E. Middieton, jr: M.M. Homobrook. Pediatrics. Vol. 47 N 5:848. mayo 1971

10 Lichtenstem and Margolits. Srience, 161:902, 1968

"Frek, O. The Ped. Clin. of. N.A., 16:95, 1969.

12 Peariaman, D. The Ped. Clin. of N.A. 16:118, 1969.

13 Carrosco, E. y col. Arch. Arq. de Alengia 10:16. 1973.

14 Soerensen, R. y col. Rev. Chil. de Ped. 44:362, 1973.

16 Brent Taylor y cols. Lancet, Vol. 2:111, 1973.

18 Hetmlich, E. The Ped. Clin. of N.A.: 16:149, 1969.

17 Szenttianyi, A.J. of Allerg., 42, 203-232, 1968.

18 Gellis Sydney, 3, Kagan, Benpamin M. "Current Pediatric Therapy", 4 Philadelphia, W.E. Saunders, 1970 .

- Smith and Parker. Proc. Central Soc. Clin. Res. 43: 76.1970.

- Mathison, D.A. JAMA, 224, 1134, 1973.

21 R. Beitrán, Oehtling. Allerg. et Inmunopat. 1:661, 1972.

22 L. Tuft, H. Muelier. "Alergia en el nin̄o", pág 228, Editorial Fediátrica, Barcelona, 1971.

2s Johnstone, D.E. and Crump, S.L. Pediatrics, 27. 39,1961

24 Johnstone, Douglas, y cals. Pediatrics, Vol. 42:793, 1968 .

26 AAS Kjell. Arch. Dis. Child. 44: $1,1969$.

28 Kenneth Mc Intosh; Elliot F. Ellis; Leonats. $S$. Hoffmon; Tillinghast. G. Lybass, ferry y Eller a.ıd Vicent A. Futginati. The Journal of Pediatrics, Vol. $82: 578,1973$.

27 Heinz Y. Wittig; C.M. Chang. Ped. Clin. of N.A. 16:55, 1969

28 Marie Brilt. Ped. Clin. of N.A., 16:227, 1969.

29 Gómez, G.; Ibánez, S. y Lama, V. Revista Chilena de Pediatría. En Prensa. 